\title{
The Effect of Silver-Plating Time on Silicon Nanowires Arrays Fabricated by Wet Chemical Etching Method
}

\author{
Shanshan Wang1, Jun Han ${ }^{1,2 *}$, Shujing Yin1 \\ ${ }^{1}$ School of Optoelectronic Engineering, Xi'an Technological University, Xi'an, China \\ ${ }^{2}$ Northwest Institutes of Advanced Technology, Xi'an Technological University, Xi'an, China \\ Email:wangshan shan@xatu.edu.cn 772366113@qq.com, *ha njun513@xatu.edu.cn
}

How to cite this paper: Wang, S.S., Han, J. and Yin, S.J. (2019) The Effect of Silver-Plating Time on Silicon Nanowires Arrays Fabricated by Wet Chemical Etching Method. Optics and Photonics Journal, 9, 1-10.

https://doi.org/10.4236/opj.2019.98B001

Received: July 12, 2019

Accepted: August 6, 2019

Published: August 9, 2019

\begin{abstract}
MACE (Metal-Assisted Chemical Etching) approach has drawn a lot of attentions due to its ability to create highly light-absorptive silicon surface. This method can generate numerous cylindrical shape microstructure on the surface of silicon like a forest, which is called "silicon nanowires arrays". This structure can dramatically suppress both reflection and transmission at the wavelength range from $400 \mathrm{~nm}$ to near-infrared $1800 \mathrm{~nm}$ by increasing the propagation path of light. In this paper, ordered silicon nanowires arrays with a large area are prepared by wet chemical etching. It is demonstrated that the SiNWs (Silicon nanowires) arrays with different morphologies can be fabricated from monocrystalline silicon of a given orientation by changing silver-plating time. Excellent anti-reflection performance in broadband wavelengths and incident angle is obtained. The fabrication method and potential application of such SiNWs in the field of photoelectric detection have great value and can provide reference for further research in this field.
\end{abstract}

\section{Keywords}

MACE, Silicon Nanowires Arrays, Anti-Reflection Performance, Photoelectric Detection

\section{Introduction}

The monocrystalline silicon has advantages of abundant reserves, mature technology, stable performance, less environmental pollution. Moreover, it is compatible with modern semiconductor technology to develop semiconductor devices to the nanometer scale. 
SiNWs are one of the most promising nanostructured materials for fabrication of high-performance devices including field effect tubes, biosensors and energy conversion device with the advantage of less front surface reflection compared to monocrystalline silicon. The preparation of SiNWs and the research of their photoelectric properties are hot topics in the field of nanotechnology [1]. Thus, the controlled growth of SiNWs is the key for potential application, such as specified length, diameter, density and electrical properties.

The SiNWs have properties of fluorescence and ultraviolet luminescence and show characteristics of high surface activity, quantum confinement benefit, field emission and electron transport. Hence, the SiNWs are applied to preparation of high-performance field effect tube, electronic detector and field emission display. With development of one-dimensional nanomaterials, SiNWs have gradually become a hot research topic.

An array of SiNWs is composed of nanowires perpendicular to the silicon substrate. The array and a single nanowire are like a forest and a tree on the silicon surface. The unique forest structure of the SiNWs arrays enable excellent characteristics of antireflection, which can result in high light absorptivity in broadband wavelengths and broad incidence angle [2]. When light is shone on such structure, it repeatedly bounces back and forth between the nanowires thus reducing surface reflection and trapping the light. The manufacturing methods mainly include CVD (Chemical Vapor Deposition) combined with ordered array technique, PLD (Pulsed Laser Deposition), physical evaporation and solvent thermal synthesis [3]. The diameter of SiNWs prepared by the aforementioned methods varies from 10 nanometers to dozens of nanometers and the length of SiNWs varies from a few microns to 20 microns. This paper introduces a new stable wet chemical etching method to fabricate silicon nanowires arrays. The relationship between morphology of SiNWs arrays and experimental parameters is investigated. The effect of morphology of SiNWs arrays on its antireflection properties is also explored. Under a specified etching condition, a uniform SiNWs arrays on the front of entire Si substrate can be obtained, even on the back. The diameter of a silicon nanowire fabricated in the laboratory is generally less than $20 \mathrm{~nm}$. The diameter can reach about $10 \mathrm{~nm}$ by strictly controlling experimental condition. This paper adopts MACE technology to improve the controllability of nanowire silicon by changing silver-plating parameters. The specific experimental steps are as follows.

\section{Experiment}

\subsection{Pretreatment of Si Wafers}

As substrate material, $525 \pm 10 \mu \mathrm{m}$ thick resistivity $(1-30 \Omega \cdot \mathrm{cm})$ p-type, $100 \pm$ $0.4 \mathrm{~mm}$ diameter, $<100>$ surface-oriented silicon wafers are used in the experiment. They are cleaned by using $\mathrm{CH}_{3} \mathrm{CH}_{2} \mathrm{OH}, \mathrm{NH}_{3} \cdot \mathrm{H}_{2} \mathrm{O}$ and $5 \% \mathrm{HF}$ solution in turn to remove inorganic pollution, dust, microorganism and other particles. The purpose is to keep the SiNWs prepared by wafer etching reaching the ex- 
pected morphology and avoid influencing their optical absorption characteristics. Each clean step requires $10 \mathrm{~min}$ of ultrasonic vibration so that the sound pressure waves parallel to the surface of the silicon wafers make the impurity particles fully soak up.

\subsection{Method}

MACE (Metal-Assisted Chemical Etching) is deposition of a layer of precious metal film (such as $\mathrm{Au}, \mathrm{Ag}, \mathrm{Pt}$, etc.) on the surface of polished $\mathrm{Si}$ substrate [4]-[9]. Then, the Sisubstrate is placed in a corrosive mixed solution consisting of HF and oxidant for a certain amount of time. The ordered columnar nanoscale forest structure is formed on the surface of $\mathrm{Si}$ substrate after chemical etching.

The aligned SiNWs are fabricated by MACE oriented p-type polished Siwafers at room temperature [10] [11]. The experimental steps are as follows. Firstly, the prewashed Si substrates are immersed into mixed solution of $4.8 \mathrm{~mol} \mathrm{HF}+0.005$ mol $\mathrm{AgNO}_{3}$ at different times, and silver plating is carried out at 100S, $120 \mathrm{~S}$, $140 \mathrm{~S}, 160 \mathrm{~S}$ and $180 \mathrm{~S}$. During this chemical reaction, the charge transfer occurs between $\mathrm{Ag}$ ions and $\mathrm{Si}$ atoms. Then, the $\mathrm{Ag}$ ions get electrons which are reduced to Ag atoms and deposited on the surface of Si wafers in the form of nanoparticles. Meanwhile, the $\mathrm{Si}$ under the $\mathrm{Ag}$ membrane is oxidized to $\mathrm{SiO}_{2}$ by $\mathrm{H}_{2} \mathrm{O}_{2}$ in the etching solution, which forms water soluble silicates under the effect of HF. As a catalyst, randomly distributed Ag nanoparticles gradually sink during the process of $\mathrm{Si}$ etching, forming an etching channel perpendicular to the surface of $\mathrm{Si}$ substrate so as to etch the entire $\mathrm{Si}$ wafers [12]. Figure 1 shows the formation mechanism of aligned SiNWs.

The deposition rate of $\mathrm{Ag}$ is much lower than the etching rate of the surrounding Si substrate. The HF is continuously etching downward along the etching channel and the coverage of Ag nanoparticles on the entire Si wafer is intermittent. Sothere are pores between adjacent Ag particles, which eventually form countless tiny columnar structure on the entire Si surface called "silicon nanowires arrays".

During the chemical reaction, the Si wafers surface form numerous chemical batteries with simultaneous cathode and anode reaction. The total reaction equation is shown below:

$$
\mathrm{Si}+2 \mathrm{H}_{2} \mathrm{O}_{2}+4 \mathrm{H}^{+}+6 \mathrm{~F}^{-} \rightarrow \mathrm{SiF}_{6}^{2-}+4 \mathrm{H}_{2} \mathrm{O}
$$

The final morphology of Si nanowires is closely related to the thickness of $\mathrm{Ag}$ membrane and the proportion of etching solution. Subsequently, the treated silicon wafers are immersed in nitric acid $\left(\mathrm{HNO}_{3}(67 \%): \mathrm{H}_{2} \mathrm{O}=1: 1\right)$ to dissolve $\mathrm{Ag}$ nanoparticles on the Si wafers surface. At last, the samples are washed with DI water and dried thoroughly in order to better observe its appearance.

\section{Experiment Results}

In order to analyze the effect of silver-plating time on the final morphology of 


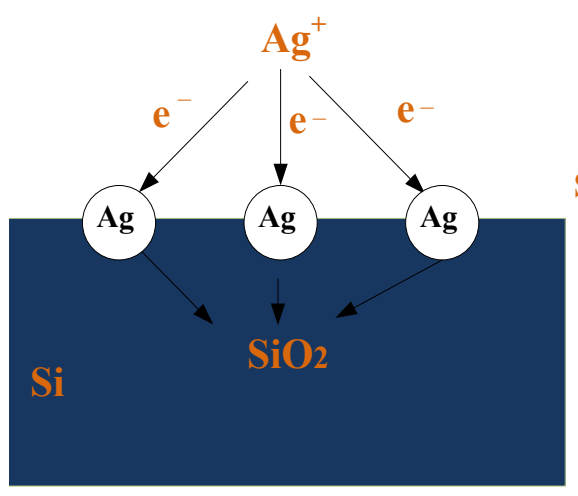

(a)

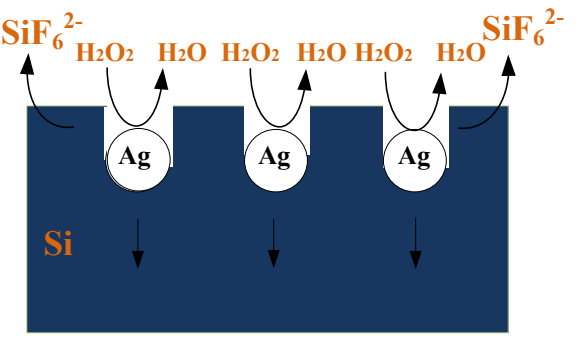

(b)

Figure 1. The formation mechanism of aligned SiNWs. (a) Ag ions are reduced to Ag atoms by getting electrons, the $\mathrm{Si}$ under the $\mathrm{Ag}$ film are oxidized to $\mathrm{SiO}_{2}$ by $\mathrm{H}_{2} \mathrm{O}_{2}$; (b) $\mathrm{SiO}_{2}$ reacts with $\mathrm{HF}$ to form water soluble silicate, while Ag nanoparticles as etching catalyst gradually sink in the direction of Si etching.

silicon nanowires, as mentioned before we put the pretreated Si wafers in to the mixed solution of $4.8 \mathrm{~mol} \mathrm{HF}+0.005 \mathrm{~mol} \mathrm{AgNO}_{3}$ in 100S, 120S, 140S, and $160 \mathrm{~S}$ respectively. A HITACHI scanning electron microscope (SEM) is employed to observe the morphological characterization of the samples. The samples are mechanically cleaved for the cross-sectional SEM investigations. The experimental result is illustrated in Figure 2.

At the initial stage of nanowires formation, $\mathrm{Si}$ is not oxidized in areas which are not covered by metal nanoparticles. The redox process in the whole system only takes place at the region of covering Ag nanoparticles, which is the key to the reaction. The location of each Ag particle determines the position of Si wafer subjected to the electrochemical corrosion, and the direction is vertical downward along the etching channel. As shown in Figure 2, thinner Ag coating facilitates formation of nanoparticles (Figures 2(a)-(c)), whereas thicker Ag coating leads to continuous Ag membrane (Figure 2(d)). The SiNWs are initially formed at the silver-plating time of $100 \mathrm{~s}$, but the morphology is irregular. For the silver-plating time of $120 \mathrm{~s}$, SiNWs are vertically downward with a certain spatial distance. As shown in Figure 2(b), the ordered nanowires form extended arrays of SiNWs with highly uniform length and diameter, which will contribute to increasing light absorptivity in broadband wavelengths. It is observed that these nanowires have the lengths of $7-11 \mu \mathrm{m}$ and sideways growth trends. The reason is that $\mathrm{Ag}$ ions getting electrons from $\mathrm{Si}$ atoms is becoming more and more difficult with growth of oxidation layer. The thickness of metal nanoparticles will not increase in the subsequent reaction and lateral growth is preferred. Huang [13] etal believe that the etching direction is mainly determined by the rate of silicon oxidation during chemical etching. The etching direction is perpendicular to the silicon substrate at high oxidation rate; It is preferred to etch along $\mathrm{Si}$ (100) direction at slow high oxidation rate. The oxidation rate is determined by the concentration of oxidation in the etching solution. For the silver-plating time of $140 \mathrm{~s}$, SiNWs are probably formed but there are significant 


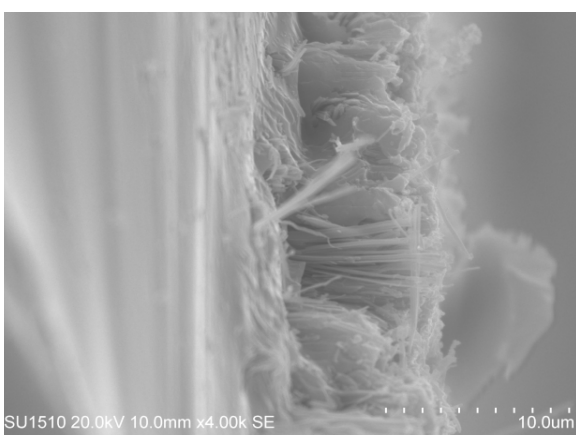

(a) Silver-plating time $100 \mathrm{~s}$

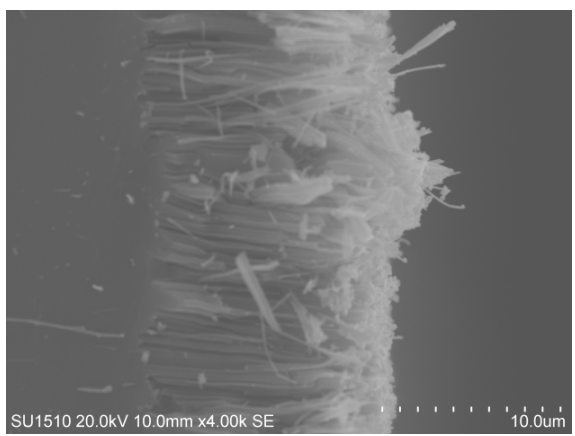

(c) Silver-plating time $140 \mathrm{~s}$

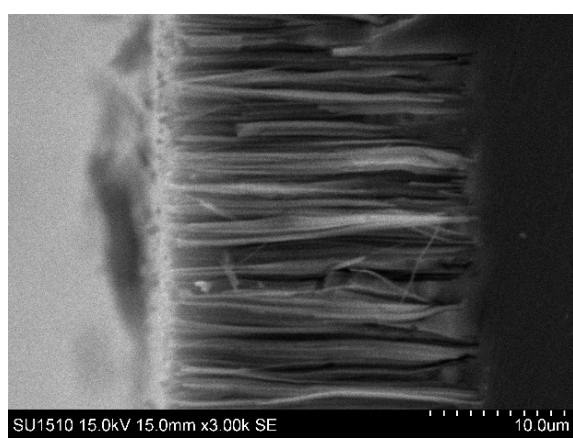

(b) Silver-plating time $120 \mathrm{~s}$

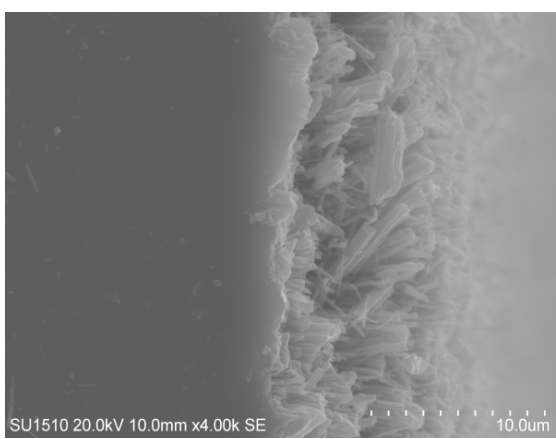

(d) Silver-plating time $160 \mathrm{~s}$

Figure 2. Cross-sectional SEM graphs of SiNWs fabricated by MACE of p-type Si substrate in a mixture solution of $\mathrm{HF}$ and $\mathrm{H}_{2} \mathrm{O}_{2}\left[\mathrm{H}_{2} \mathrm{O}_{2}: \mathrm{HF}: \mathrm{H}_{2} \mathrm{O}=5\right.$ :12:37] for $40 \mathrm{~min}$ at room temperature.

fractures of nanowires. The forest structure of nanowires is disordered and no linear pattern can be formed in the silver-plating time of $160 \mathrm{~s}$. For comparison, the thickness of Ag coating covering Si wafers can clarify the final morphology of SiNWs.

The longer the silver-plating time is, the density of Ag particles will increase, and the less Si wafers area etched by HF will be provided. This will lead to an increase in porosity of the whole forest structure. As shown is Figure 3(d), the Ag particles density deposited on the surface of Si wafers is the highest of four samples due to the excess Ag atoms produced in the reduction reaction. There is slight chemical reaction with HF in remaining areas not covered by $\mathrm{Ag}$, and the SiNWs produced have smaller diameter and nonuniform lengths which leads to fracture easily. In the case of silver-plating time of 100s (Figure 3(a)), the density of Ag particles on the Si substrate decreases. The location of each Ag particle determines the position of $\mathrm{Si}$ wafer corroded by electrochemistry. From the top view of SiNWs, there are many thin lamellar structures on the surface. The experimental parameters of Figure $\mathbf{3}$ (b) present a uniform and regular columnar forest structure. When the light is shone on such a dense forest structure, it repeatedly bounces back and forth between the nanowires to be more effective on antireflection. This capability to have a low reflectivity make the structure possess a wide range of applications including infrared detectors, biological sensors and solar cells. 


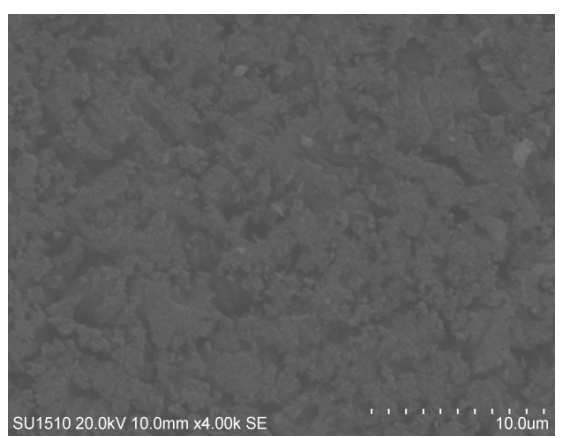

(a) Silver-plating time $100 \mathrm{~s}$

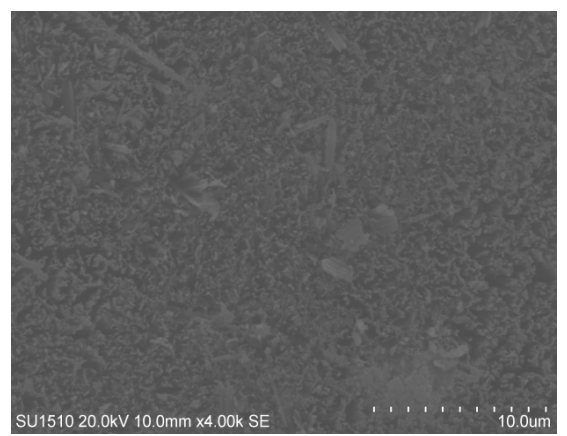

(c) Silver-plating time $140 \mathrm{~s}$

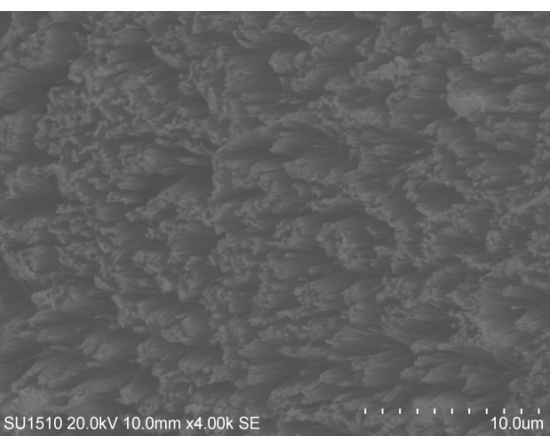

(b) Silver-plating time $120 \mathrm{~s}$

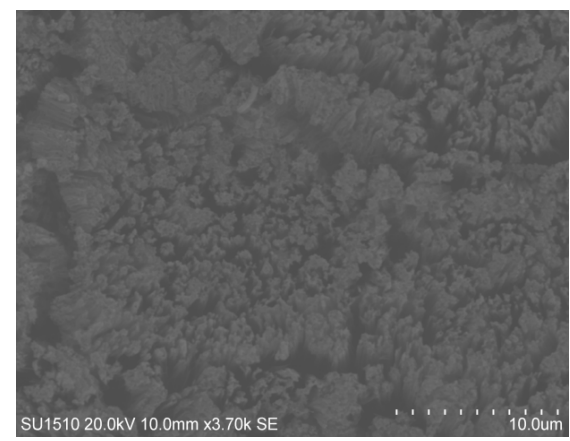

(d) Silver-plating time $160 \mathrm{~s}$

Figure 3. Top view ofSEM images of SiNWs fabricated by MACE.

\section{Discussion}

In order to check optical properties of nanowires on the silicon surface, the entire transmittance at a wavelength range from 250 to $1800 \mathrm{~nm}$ is measured by UV/VIS/NIR Spectrometer Perkin-Elmer Lambda 950 equipped with an integrating sphere. The principle of the integrating sphere is that a certain wavelength of light through the sampling port is collected, homogenous scattering within the integrating sphere after multiple reflection. It is reliable to use integrating sphere for measuring the luminous flux.

The uniform density of SiNWs arrays in the forest structure will result in multiple scattering of incident light between silicon wires, which greatly extends the path of light propagation and enhance the trap effect of incident light. The silver-plating time is directly related to the density of silicon nanowires. Figure 4 shows the comparison of optical properties of silicon nanowires arrays with different silver-plating time.

One of the most important parameters of light absorption is the transmittance as function of wavelength. The light source is perpendicularly illuminated on the samples in order to get the same reflectivity. The three samples have been depicted in Figure 4(a): they show the same trend in the visible light and near-infrared spectral regions with a wavelength of $380 \mathrm{~nm}-1000 \mathrm{~nm}$. The transmittance of monocrystalline silicon is higher than the others, and the other two samples show consistent changes at the wavelength of nearly $1000 \mathrm{~nm}$ - 


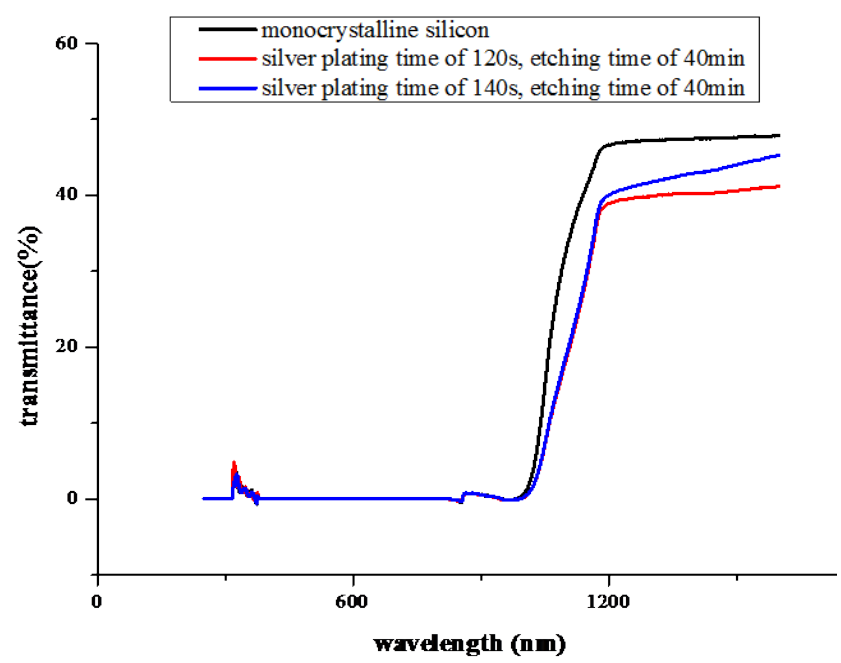

(a)

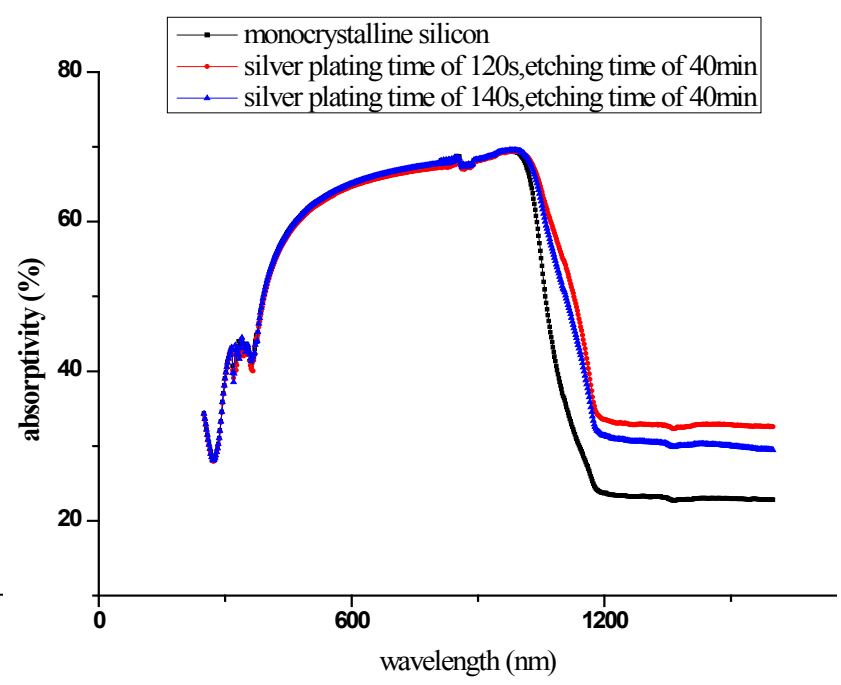

(b)

Figure 4. The comparison of optical properties of silicon nanowires with different silver-plating time. (a) The transmittance graph of three samples; (b) The absorptivity graph of three samples.

$1200 \mathrm{~nm}$. When the wavelength is beyond $1200 \mathrm{~nm}$, the transmittance of silver-plating $140 \mathrm{~s}$ sample increases from $38 \%$ to $45 \%$ and the curve of silver-plating $120 \mathrm{~s}$ is almost unchanged. The sample with silver-plating $120 \mathrm{~s}$ performs the best in capturing light, especially at wavelength beyond $1200 \mathrm{~nm}$. However, the sample with silver-plating $140 \mathrm{~s}$ also shows a low transmittance, and even just a little higher at the wavelength of $1000 \mathrm{~nm}-1200 \mathrm{~nm}$ compare with red line. This indicates that the nanowires have been formed. The transmittance increases at wavelength beyond $1200 \mathrm{~nm}$ due to larger interline pores and partial lack of dense forest structure.

Silicon nanowires membrane not only suppresses light transmittance, but also increases absorptivity of substrate to the incident light in broadband wavelengths. Previous researchers believe that the bandwidth absorption characteristic is caused by the resonance of incident light waves in the silicon wires arrays. Garnet and Yang [14] measure the transmission and photocurrent of silicon nanowires arrays under the AM $1.5 \mathrm{G}$ spectrum, and found that the propagation path of light increased by more than 70 times. Muskens [15] et al. studies the optical scattering in the growing disordered silicon nanowires arrays and get a conclusion that the nanowires arrays can effectively couple the incident light into the forest structure through multiple scattering. At the California Institute of Technology, the Harry Atwater group [16] use VLS method to prepare the SiNWs. Their experimental result shows this kind of multiple trapping structure can significantly improve the light absorption of infrared band, compared with planar silicon of ideal back-reflector and optical texture, which is consistent with our experimental result.

Huang Haihua, Zhang Weiping et al. [17] of Guangxi University have expressed their views on the absorptivity of cylindrical silicon nanowires arrays. They assume that the background medium of silicon nanowires is air. The 3D 
sketch of the simulated surface of SiNWs is shown in Figure 5.

Where $\mathrm{P}$ is the period of arrays, $\mathrm{D}$ and $\mathrm{H}$ are the diameter and the height of nanowire, respectively. The duty ratio is $f=\mathrm{D} / \mathrm{P} . \mathrm{D}$ is assumed to be a constant. The absorption efficiency corresponding to different duty ratio $f$ is obtained through the calculation formula [18]. As mentioned above, the longer the silver-plating time is, the density of Ag particles on Si substrate will increase, which will lead to an increase in porosity of the whole forest structure. It means that the period of arrays $\mathrm{P}$ increases, the duty ratio $\mathrm{f}$ bound to drop a lot. As shown in Figure 4(b), when the wavelength is less than $800 \mathrm{~nm}$, the blue line is just a little higher than the red line. The red line is significantly higher than blue line at wavelength beyond $1100 \mathrm{~nm}$. In this case, $f$ is not proportional to the absorption efficiency and it is directly related to the wavelength [18]. As shown in Figure 6, when $f<0.8$, the absorption efficiency increases with increasing $f$, when $f>0.8$, it is just the opposite. Our experimental results show that $f$ is less than 0.8 , which belongs to the former but we need to consider the wavelength range. $\mathrm{Hu} \mathrm{L}$ and Chen G [19] [20] analyzes the effects of nanowire diameter, length, and duty ratio on optical properties of periodic Si nanowires arrays. They point out that the reflectivity of nanowires is significantly lower than a planar silicon in the wide spectral range due to the reduction of nanowire density in a certain condition.

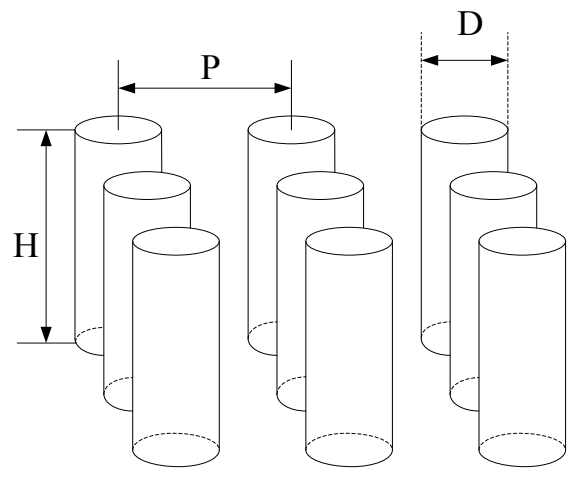

Figure 5. 3D sketch of the simulated surface.

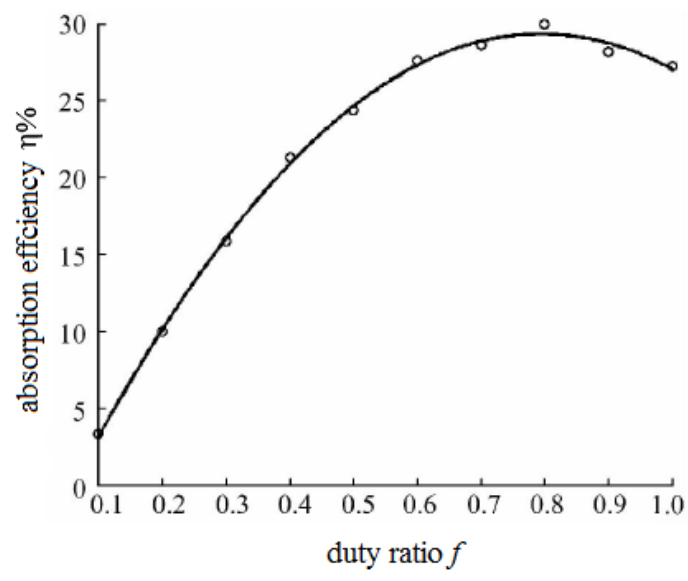

Figure 6. The relationship of absorption efficiency and duty ratio. 


\section{Conclusion}

We have demonstrated metal-assisted chemical etching to fabricate silicon nanowires arrays at room temperature by changing experimental parameters. The aligned silicon nanowires on the silicon substrate with 7 - $11 \mu \mathrm{m}$ length forming by forest structure can be generated if the parameters are properly controlled. Furthermore, we confirm that the optimized $\mathrm{Ag}$ deposition forms a layer of membrane as etching catalyst to coordinate the density of the nanowires, which has exceptional performance on antireflection. The transmittance of the forest structured surface with silver-plating $120 \mathrm{~s}$ sample is around $38 \%$ with spectral response beyond $1200 \mathrm{~nm}$, which is much lower than that of the other two samples. In addition to silver-plating time, the forest morphology of SiNWs is also affected by other experimental parameters, such as the solution concentration, temperature in metal-assisted chemical etching, and the etching time. The discussion about the effect of such parameters will be provided in the further with more experiments.

\section{Acknowledgements}

We acknowledge support from the key Laboratory Program of Shaanxi Province.

\section{Conflicts of Interest}

The authors declare no conflicts of interest regarding the publication of this paper.

\section{References}

[1] Kelzenberg, M.D., Boettcher, S.W., Petykiewicz, J.A., et al. (2010) Enhanced Absorption and Carrier Collection in Si Wire Arrays for Photovoltaic Applications. Nature Materials, 9, 239-244. https://doi.org/10.1038/nmat2635

[2] Krc, J., Zeman, M., Smolc, F. and Topic, M. (2004) Optical Modelling of Thin-Film Silicon Solar Cells Deposited on Textured Substrates. Thin Solid Films, 451-452, 298-302. https://doi.org/10.1016/j.tsf.2003.11.030

[3] Wang, Y.B. (2012) The Synthetize and Optical Properties Research of Silicon Nanowires by the Wet Etching. Changchun University of Science and Technology, 1-5.

[4] Wang, B. (2010) Silicon Nanowires Prepared by Wet Chemical Etching Method Northeast Normal University, 9-11.

[5] Besma, M. and Abdelkader, B.J. (2017) Correlation between Oxidant Concentrations, Morphological Aspects and Etching Kinetics of Silicon Nanowires during Silver-Assist Electroless Etching. Applied Surface Science, 425. https://doi.org/10.1016/j.apsusc.2017.06.110

[6] Mei, C.L., Liu, S., Huang, X., et al. (2017) Localized Surface Plasmon Induced Position-Sensitive Photodetection in Silicon-Nanowire-Modified Ag/Si. Small, 13. https://doi.org/10.1002/smll.201770222

[7] Rahman, T. and Boden, S.A. (2017) Optical Modeling of Black Silicon for Solar Cells Using Effective Index Techniques. IEEE Journal of Photovoltaics, 7, 1556-1562. https://doi.org/10.1109/jphotov.2017.2748900

[8] Liao, C.J., Yang, P.Z., Liao, H., et al. (2013) Investigation on the Mechanism of Met- 
al-assisted Chemical Etching Used for Black Silicon Preparation. Journal of Yunnan Normal University, 33, 22-28.

[9] Yu, D. (2015) Study of Cu-Assisted Chemical Etching for Fabricating Nano-Structured Silicon. Collaborative Innovation Center of Advanced Microstructure, Lab of SolidState Microstructure, School of Physics, Nanjing University, 13-18.

[10] Chen, C.Y., Wu, C.S., Chou, C.J., et al. (2010) Morphological Control of Single-Crystalline Silicon Nanowire Arrays near Room Temperature. Advanced Materials, 20, 3811-3815. https://doi.org/10.1002/adma.200702788

[11] Chartier, C., Bastide, S. and Lévy-Clément, C. (2008) Metal-Assisted Chemical Etching of Silicon in $\mathrm{HF}-\mathrm{H}_{2} \mathrm{O}_{2}$. Electrochemical Acta, 53, 5509-5516. https://doi.org/10.1016/j.electacta.2008.03.009

[12] Dřínek, V., Klementová, M., Fajgar, R. and Dytrych, P. (2015) Silicon Nanowires Grown on Metal Substrates via Self-Catalyst Mechanism. Materials Letters, 160, 109-112. https://doi.org/10.1016/j.matlet.2015.07.098

[13] Huang, Z.P., Shimizu, T.H., Senz, S., Zhang, Z., Geyer, N. and Gosele, U. (2010) Oxidation Rate Effect on the Direction of Metal-Assisted Chemical and Electrochemical Etching of Silicon. Chem. C, 114, 10683-10690.

https://doi.org/10.1021/jp911121q

[14] Garnett, E. and Yang, P.D. (2010) Light Trapping in Silicon Nanowire Solar Cells. Nano Lett, 10, 1082-1087. https://doi.org/10.1021/nl100161z

[15] Muskens, O.L., Rivas, J.G., Algra, R.E., et al. (2008) Lagendijk. Design of Light Scattering in Nanowire Material for Photovoltaic Applications. Nano Letters, 8, 2638-2642. https://doi.org/10.1021/nl0808076

[16] Kelzenberg, M.D., Boettcher, S.W., Petykiewicz, J.A., Turner-Evans, D.B., Putnam, M.C., Warren, E.L., Spurgeon, J.M., Briggs, R.M., Lewis, N.S. and Atwater, H.A. (2010) Enhanced Absorption and Carrier Collection in Si Wire Arrays for Photovoltaic Applications. Nature Materials, 9, 239-244. https://doi.org/10.1038/nmat2635

[17] Huang, H.H., Zhang, W.P., Xiao, Y.F., et al. (2013) The Effect of the Nanowire Shape on the Optical Absorption of the Silicon Nanowire Film Solar Cells. Journal of Guangxi University: Nat Sci Ed., 38, 777-783.

[18] Lin, C.X. and Povinelli, M.L. (2009) Optical Absorption Enhancement in Silicon Nanowire Arrays with Alarge Lattice Constant for Photovoltaic Applications. Optics Express, 17, 19371-19381. https://doi.org/10.1364/oe.17.019371

[19] Lagos, N., Sigalas, M.M. and Niarchos, D. (2011) The Optical Absorption of Nanowire Arrays. Photonics and Nano Structures Fundamental and Applications, 9, 163-167. https://doi.org/10.1016/j.photonics.2010.09.005

[20] Hu, L. and Chen, G. (2007) Analysis of Optical Absorption in Silicon Nanowire Arrays for Photovoltaic Application. Nano Letters, 7, 3249-3252. https://doi.org/10.1021/nl071018b 